\section{Low incidence of clinically relevant bleeding complica- tions after fast-track arthroplasty: a register study of 8,511 arthroplasties}

\author{
Annette MOISANDER ${ }^{1}$, Konsta PAMILO ${ }^{2}$, Antti ESKELINEN ${ }^{2,3}$, Jukka HUOPIO ${ }^{4}$, \\ Hannu KAUTIAINEN ${ }^{5}$, Anne KUITUNEN ${ }^{6}$, Pirjo MUSTONEN ${ }^{7}$, and Juha PALONEVA ${ }^{8}$
}

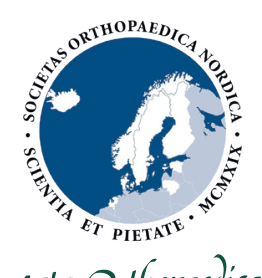

Acta Orthopaedica

\footnotetext{
${ }^{1}$ Department of Anesthesia and Intensive Care, Hospital Nova, Central Finland Healthcare District, Jyväskylä; ${ }^{2}$ Coxa Hospital for Joint Replacement, Tampere; ${ }^{3}$ Faculty of Medicine and Health Technologies, University of Tampere, Tampere; ${ }^{4}$ Department of Orthopaedics and Traumatology, University Hospital, Kuopio; ${ }^{5}$ Primary Health Care Unit, Kuopio University Hospital, Finland, Folkhälsan Research Center, Helsinki; ${ }^{6}$ Department of Intensive Care, University of Tampere, Tampere University Hospital, Tampere; ${ }^{7}$ Turku University Hospital, Turku; ${ }^{8}$ Department of Surgery, Hospital Nova, Central Finland Healthcare District, Jyväskylä, Finland

Correspondence: annette.moisander@ksshp.fi

Submitted 2021-12-27. Accepted 2022-02-04.
}

Background and purpose - Fast-track total joint replacement (TJR) has become increasingly common. Routine thromboprophylaxis for pulmonary embolism and deep venous thrombosis prevention lasts from 2 to 5 weeks. This retrospective registry study focused on clinically relevant bleeding complications 90 days after fast-track primary TJR.

Patients and methods - All primary fast-track total hip (THA) and knee arthroplasties (TKA) performed between 2015 and 2016 were extracted from the Finnish Arthroplasty Register and Finnish Hospital Discharge Register. Type of arthroplasty and indication for the operation were combined with diagnoses of clinically relevant bleeding complications within 90 days of surgery. The incidence of these bleedings was the primary outcome measure.

Results - Of the total of 8,511 patients (mean age 67 years (SD 10); 60\% female), $45 \%$ underwent unilateral THA, 52\% unilateral TKA, and 3\% bilateral TKA. The incidence of clinically relevant bleeding complications within 90 days was $1 \%$ (95\% CI $0.8-1.3$ ). No difference was observed in bleeding incidence between the groups. The 87 bleedings comprised 57 operative site bleedings, 17 gastrointestinal bleedings, 6 intracranial non-traumatic bleedings, 5 bleedings from the nose or another undetermined site, and 2 intraocular bleedings. 1 death due to intracranial bleeding was recorded, and hence clinically relevant bleeding-specific 90-day mortality was $0.01 \%$.

Interpretation - The incidence of clinically relevant bleeding complications was low. However, they cause patient discomfort, increase the use of healthcare services, and can be life-threatening and even fatal.
Risk factors contributing to postoperative bleedings include advanced age, use of non-steroidal anti-inflammatory drugs, glucocorticoids, antiplatelet medications, and thromboprophylaxis to prevent venous thromboembolisms (VTE) (1-3).

The incidence of bleeding has been reported to range from $1 \%$ to $3 \%$ in arthroplasty patients receiving thromboprophylaxis $(4,5)$. Use of thromboprophylaxis provokes and potentially maintains bleeding. Before fast-tracking focusing on reducing complications, $15-30 \%$ of arthroplasty patients experienced VTE (4). Thromboprophylaxis, better surgical and anesthesia techniques, and earlier mobilization have decreased the incidence of VTE to $1 \%$ and lower (4,6-8).

Owing to variation across studies in the definitions of bleedings, it is difficult to compare incidence rates (9). The European Medicines Agency has provided definitions for major bleedings for research on the use of VTE prophylaxis in surgical patients. Major bleedings are defined as fatal bleeding, major symptomatic bleeding (intracranial, intra- or retroperitoneal, intraocular, intramuscular leading to compartment syndrome), extra-surgical bleeding causing a decrease in hemoglobin of at least $20 \mathrm{~g} / \mathrm{L}$, bleeding at the operative site leading to intervention or delaying rehabilitation or wound healing, unexpected or prolonged bleeding, and surgeon-assessed bleeding sufficient to cause hemodynamic instability. To be classified as major, these bleedings should also cause a fall in hemoglobin of at least $20 \mathrm{~g} / \mathrm{L}$ or require at least 2 units of blood transfusion $(\mathbf{1 0 , 1 1 )}$.

As data on bleeding complications in fast-track total joint replacement is scarce, we conducted a retrospective registry study using information from the Finnish Arthroplasty Regis- 
ter and Finnish Hospital Discharge Register to determine the incidence of clinically relevant bleeding complications related to fast-track arthroplasty.

\section{Patients and methods}

This study was a retrospective register-based study of unselected consecutive THA and TKA patients operated using a fast-track protocol in 3 publicly funded academic hospitals in Finland (Kuopio University Hospital, Kuopio; Coxa Hospital for Joint Replacement, Tampere; and Hospital Nova, Jyväskylä). During the study period, the catchment area of these hospitals contained a population of $1,713,738$, or $31 \%$ of the Finnish population (12).

Fast-track arthroplasty aims at better clinical outcomes, fewer complications, and a shorter hospital stay. All patients receive preoperative information on the standardized treatment protocol, including opioid-sparing pain relief, early mobilization, and discharge criteria. Anesthetic methods include low-dose opioid-free spinal anesthesia or total intravenous anesthesia, and local anesthesia, which is used intraoperatively and postoperatively to ensure pain relief. Use of catheters and drains is avoided. Patients are mobilized on the day of surgery and thromboprophylaxis is started within 6-8 hours after surgery. Patients are discharged, preferably to their home, when the relevant criteria are met (13). In Finland, the length of pharmacological thromboprophylaxis with low molecular weight heparin or direct oral anticoagulants is $28-35$ and $10-14$ days for THA and TKA, respectively (14).

We identified all arthroplasties from the Finnish Arthroplasty Registry (FAR) between January 1, 2015, and December 31, 2016. Operations were identified by the NOMESCO (15) codes for primary THA: NFB30, NFB40, NFB50, NFB60, and NFB99, and for primary TKA: NGB20, NGB30, NGB40, and NGB50. Patients under 18 years of age were excluded. We included all patients with or without permanent anticoagulation. Patient age and sex and the indication for TJA were obtained from FAR.

All the study hospitals had established a fast-track protocol by the year 2015. All patients received information on the protocol, which was aimed at early discharge home. This information included early mobilization, discharge criteria, and pain management. Pain management was multimodal and local infiltration anesthetic techniques were used with TKA patients to avoid excessive opioid use. Multimodal pain management included combinations of paracetamol, non-steroidal anti-inflammatory drugs, COX-2 selective pain medication, or gabapentinoids. Surgery was performed under opioidfree low-dose spinal anesthesia. Intraoperative use of drains and catheters was avoided, and intravenous tranexamic acid was administered. Discharge to ward after discharge criteria from post-anesthesia care unit were met, where patients were mobilized on the day of the surgery. Thromboprophylaxis was started 6-8 hours after surgery. Patients were discharged home as soon as the discharge criteria were met.

These data were then combined with data from the Finnish Hospital Discharge Register (FHDR) to identify clinically relevant non-traumatic bleedings by their ICD-10 diagnosis codes, 10th revision (16). These clinically relevant bleedings follow the definition of major bleedings published in studies of thromboprophylaxis in surgical patients (11). Data on blood transfusions are not included in this register. Hence, all bleedings recorded in the FHDR were assumed to be clinically relevant. We also conducted a preliminary survey of all the ICD-10 codes recorded in the research population to identify all non-traumatic bleedings.

The FHDR and FAR are both maintained by the National Institute for Health and Welfare. It is mandatory for all Finnish hospitals to report all surgical procedures to the FHDR. The FAR data include all hip and knee implants operated on in Finland from 1980 onwards (17). Dates of death were obtained from the Population Information System maintained by the Population Register Centre. Causes of death were obtained from Statistics Finland.

From this combined data we calculated the length of stay (LOS) and the length of uninterrupted institutional care (LUIC). LOS was counted as the number of nights in the surgical specialty ward of the hospital terminating in either discharge home or transfer to another facility. LUIC was calculated as the number of nights spent in care facilities before postoperative discharge home. Patients using permanent anticoagulation receive reimbursement from the Finnish Social Insurance Institution. All patients eligible for compensation were identified, to have an idea of the scale of these patients among study population.

Patients were then grouped according to operation type. Due to the low number (under 50) of bilateral hip arthroplasties performed under the same anesthesia, these operations were excluded from the further analyses.

\section{Statistics}

Descriptive statistics are presented as means (SD), as medians (IQR), or as counts (\%). Bleeding incidences were estimated by an exact $95 \%$ confidence interval (CI) based on a Poisson distribution. Time-to-event analysis was based on the KaplanMeier failure function. Stata 15.1 (StataCorp LP, College Station, TX, USA) was used for the analysis.

\section{Ethics, registration, funding, and potential conflicts of interest}

Permission for the study was obtained from the National Institute for Health and Welfare, the Finnish Social Insurance Institution, and Statistics Finland separately. According to Finnish legislation, ethical permission was not required to perform this registry study. AM received a grant from the Finnish Medical Research Foundation and from the Finnish Arthro- 
Table 1. Basic demographics of fast-track total joint arthroplasty (TJA) patients and clinically relevant bleeding complications. Values are count (\%) unless otherwise specified

\begin{tabular}{|c|c|c|c|c|}
\hline Factor & $\begin{array}{l}\text { Total hip } \\
\text { arthroplasty }\end{array}$ & $\begin{array}{l}\text { Total knee art } \\
\text { unialteral }\end{array}$ & $\begin{array}{l}\text { rthroplasty } \\
\text { bilateral } \mathrm{C}\end{array}$ & Combined (\%) \\
\hline Count & $3,831(45)$ & 4,394 (52) & $286(3)$ & $8,511(100)$ \\
\hline Mean age (SD) & $67(11)$ & $68(10)$ & 64 (8) & $67(10)$ \\
\hline \multicolumn{5}{|l|}{ Sex } \\
\hline Male & 1,694 & 1,538 & 144 & $3,376(40)$ \\
\hline Female & 2,137 & 2,856 & 142 & $5,135(60)$ \\
\hline \multicolumn{5}{|l|}{ Diagnosis } \\
\hline Osteoarthritis & 3,216 & 3,690 & 240 & 7,146 (84) \\
\hline \multicolumn{5}{|l|}{ Rheumatoid } \\
\hline arthritis & 87 & 99 & 7 & $193(2)$ \\
\hline Other & 528 & 605 & 39 & $1,172(14)$ \\
\hline \multicolumn{5}{|c|}{ Clinically relevant bleeding complications } \\
\hline Count & $38(44)$ & $46(53)$ & $3(3)$ & $87(100)$ \\
\hline $\begin{array}{l}\text { Incidence, \% } \\
(95 \% \mathrm{Cl})\end{array}$ & $\begin{array}{l}1.1 \\
(0.7-1.4)\end{array}$ & $\begin{array}{l}1.1 \\
(0.8-1.4)\end{array}$ & $\begin{array}{l}1.1 \\
(0.3-3.2\end{array}$ & $\begin{array}{ll} & 1.0 \\
2) \quad(0.8-1.3)\end{array}$ \\
\hline
\end{tabular}

plasty Society to conduct this study. No conflicts of interest were declared.

\section{Results}

Between 2015 and 2016, 8511 fast-track arthroplasty operations were performed in the participating hospitals. The data included 3831 THAs, 4394 TKAs, and 286 bilateral TKAs (operated on under the same anesthesia) (Table 1). The data covered approximately $20 \%$ of all primary TJAs performed in Finland over the study period $(\mathbf{1 8 , 1 9 )}$. Of all 8511 TJA patients, $487(5.7 \%)$ had permanent anticoagulation. Mean length of stay (LOS) was 3 (SD 1) days and mean length of uninterrupted institutional care (LUIC) after the operation was 4 (SD 1) days.

The incidence of clinically relevant bleeding complications was $1 \%$ (CI $0.8-1.3$ ) within 90 days after the primary fast-track TJA (Figure). As shown in Table 1, the incidence of clinically relevant bleeding complications did not vary between the 3 operation types. The 87 bleedings consisted of 57 operation site bleedings, 17 gastrointestinal bleedings, 6 spontaneous intracerebral bleedings, 5 bleedings elsewhere, and 2 intraocular bleedings (Tables 2 and 3). A little over half of the bleedings occurred in the TKA patients. Patients with permanent anticoagulation accounted for $5(5.7 \%)$ of the 87 with clinically relevant bleeding complications.

The median time to clinically relevant bleeding complications was 14 days. It was shortest for intraocular bleeding, followed by operational site bleeding, gastrointestinal bleeding, non-traumatic intracranial bleeding, and bleeding elsewhere. 8 clinically relevant bleeding complications were found within 5 days of TJA: these comprised 4 operational site bleedings, 2 intraocular bleedings, 1 gastrointestinal bleeding, and 1 bleeding elsewhere.
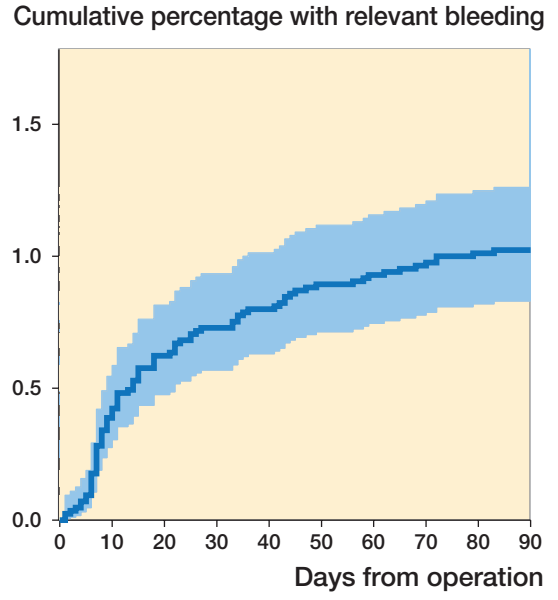

Timeline for clinically relevant bleeding complications with $95 \% \mathrm{Cl}$

Table 2. Patient demographics according to clinically relevant bleeding complications. Values are count $(\%)$ unless otherwise specified

\begin{tabular}{lcc}
\hline & Clinically relevant bleeding & complications \\
No & Yes \\
Factor & $\mathrm{n}=8,424$ & $\mathrm{n}=87$ \\
\hline Women & $5,087(60)$ & $48(55)$ \\
Mean age (SD) & $67(10)$ & $69(10)$ \\
Diagnosis & & \\
$\quad$ Osteoarthritis & $7,078(84)$ & $68(78)$ \\
$\quad$ Rheumatoid arthritis & $193(2)$ & 0 \\
$\quad$ Other & $1,153(14)$ & $19(22)$ \\
Procedure & & \\
$\quad$ Total hip arthroplasty & $3,793(45)$ & $38(44)$ \\
$\quad$ Total knee arthroplasty & $4,348(52)$ & $46(53)$ \\
$\quad$ Bilateral total knee arthroplasty & $83(3)$ & $3(3)$ \\
Death within 90 days from surgery & $33(0.4)$ & $2(2)$ \\
\hline
\end{tabular}

Table 3. Clinically relevant bleeding complications and presentation time from operation

\begin{tabular}{lcc}
\hline Bleeding site & $\begin{array}{c}\text { Female/Male } \\
\mathrm{n}\end{array}$ & $\begin{array}{c}\text { Presentation time } \\
\text { days, median (range) }\end{array}$ \\
\hline Operation site & $27 / 30$ & $10(1-72)$ \\
Gastrointestinal & $13 / 4$ & $34(1-83)$ \\
Non-traumatic intracranial & $5 / 1$ & $37(21-72)$ \\
Elsewhere & $2 / 3$ & $44(5-79)$ \\
Intraocular & $1 / 1$ & $2(1-3)$ \\
All together & $48 / 39$ & $14(1-83)$ \\
\hline
\end{tabular}

There were 35 deaths within 90 days after the index operation, indicating a 90-day mortality of $0.4 \% .2$ occurred in the group of bleeding patients, 1 of which was caused by spontaneous intracerebral bleeding 53 days after TJA. The other death was not due to bleeding. Thus, clinically relevant bleeding-specific 90 -day mortality was $0.01 \%$. 


\section{Discussion}

In our study, the incidence of clinically relevant bleeding complications 90 days after primary fast-track TJA was $1 \%$. The incidences among THA, TKA, and BTKA were similar.

Reports of bleedings associated with fast-track arthroplasty are scarce. In a French study the incidence of bleeding complications was $1.7 \%$ (5) and no difference was found between THA and TKA. The difference in incidence between the 2 studies is partly due to a difference in the inclusion criteria: Jenny et al. (5) included all patients requiring blood transfusion for any reason.

Bleedings in arthroplasty patients have mainly been reported in thromboprophylaxis studies. The incidence of bleedings reported in a review of thromboprophylaxis studies varied between $0.1 \%$ and $3.1 \%$ for THA, and $0.2 \%$ and $1.4 \%$ for TKA (9). None of these studies included fast-track patients. The variation in incidences is due to the lack of a consensus on the definition of bleedings, and to variation in both the duration of follow-ups and the type of thromboprophylaxis used.

The European Medicines Agency (EMA) definition of major bleeding recommended for use in clinical investigations of antihemostatic medical products in surgical patients cannot be fully followed in a registry study of this kind. First, this guideline was introduced to study new antihemostatic drugs, preferably in blinded randomized control trials, and not for registry studies. Second, most measurements of operative site bleedings have been subjective reports by the surgeon and thus cannot be defined based on hospital discharge registers. However, we used the EMA definition as far as this was possible regarding the study type. Our study included all recorded fatal bleedings and bleedings in critical organs and all bleedings recorded in the hospitals' discharge registers, since to be recorded in this type of register means that they must had been considered clinically relevant.

The most common bleedings in our study were operative site bleedings, which accounted for two-thirds of all the clinically relevant bleedings. Operative site bleedings were also included in Jenny et al. but have rarely been reported in many other studies on bleeding after TJA $(5,9)$. While not life threatening, these bleedings cause patient discomfort, pain, distress, and inconvenience. They may also increase the risk of superficial and deep infections. Although approximately $27 \%$ of septic infections after TJA have been associated with surgical site infection, the causality of surgical site bleedings and postoperative prosthetic joint infections remains unclear $(\mathbf{2 0 , 2 1 )}$.

All arthroplasty patients have at least 1 of the following risk factors for gastrointestinal bleeding (GIB): old age, stress due to the operation, and use of non-steroidal pain medication or glucocorticoids. In a previous study, the incidence of gastrointestinal ulcer among primary fast-track TJA patients was $0.5 \%$ (22). As not all ulcers bleed, the true incidence is probably higher.
To identify and treat gastrointestinal bleedings usually requires an invasive procedure and hence readmission. Studies of Finnish fast-track patients from 2012 to 2013 reported readmission rates of $0.2 \%$ and $0 \%$ for GIB within 42 days of THA and TKA, respectively $(\mathbf{1 3 , 2 3 )}$. A slightly higher incidence of $0.6 \%$ readmissions for all gastrointestinal complications was recently reported for a Finnish fast-track TJA hospital (24). While gastrointestinal bleedings in fast-track arthroplasty patients are rare, they increase costs due to the need for readmission, invasive procedures, and blood transfusions. The presence of potent anticoagulation increases the risk of lifethreatening gastrointestinal bleeding.

Non-traumatic intracranial hemorrhage is a potentially fatal bleeding complication. Up to $40 \%$ of these patients die within a month and under $40 \%$ achieve functional independence (25). We found 2 deaths; 1 was due to an intracerebral bleeding. The other was not due to bleeding and thus the clinically relevant bleeding complication-specific 90-day mortality rate was $0.01 \%$. Berg et al. (22) in their study on cerebrovascular events in Swedish fast-track arthroplasty patients, including both bleeding and thrombosis, reported an incidence of $0.4 \%$ for both THA and TKA.

Blood loss and bleeding after arthroplasty can be minimized in several ways, for example by intravenous and intra-articular tranexamic acid (TXA) (26). Although the exact amount of TXA administered cannot be known in a register study of this kind, we do know that at least 1 dose of intravenous TXA (1 g) was administered during surgery and that no intra-articular dosing was used. The short-term use of TXA affects both operative site bleeding and blood loss for a few days after the operation. All study hospitals used compression bandages for total knee arthroplasties to reduce postoperative wound bleeding.

Use of thromboprophylaxis may prolong existing bleeding or predispose to bleeding by complicating the management of bleeding. In the absence of thromboprophylaxis and fasttracking, at least $30 \%$ of TJA patients experience a venous thromboembolism or pulmonary embolism (4). Regular use of thromboprophylaxis has diminished the incidence of VTEs to $1-2 \%$ (4). Studies on venous thromboembolic complications in fast-track TJA patients have reported a much lower incidence (5-8). Husted et al. (6) reported 90-day incidences of symptomatic deep vein embolism and pulmonary embolism to be $0.65 \%$ and $0.30 \%$, respectively, in fast-track TJA patients who had only received thromboprophylaxis during hospitalization. Although in their study the length of hospitalization decreased, meaning shorter duration of thromboprophylaxis, the authors found no increase in the incidences of venous embolisms.

Jørgensen et al. reported an incidence of venous thromboembolism of $0.41 \%$ within 90 days of surgery (7). This study population was subsequently included in a larger study of 17 582 fast-track TJA patients, where the incidence of symptomatic deep vein and pulmonary embolism was found to be $0.4 \%$ 
(8). The patients in these studies received thromboprophylaxis only during hospitalization, providing it was 5 days or less. The median length of hospitalization was 2 days. This low incidence of VTE after fast-track TJA indicates a possible potential for more individualized scheduled thromboprophylaxis $(5,27)$

The length of thromboprophylaxis needed after TJA and especially after fast-track TJA has been questioned (28). The Finnish thromboprophylaxis recommendation does not differ between care protocols. Our study showed that the use of longer thromboprophylaxis does not lead to the excessive occurrence of bleeding complications in Finnish fast-track arthroplasty patients. However, it raises the question: Might the incidence of clinically relevant bleeding complications be even lower if thromboprophylaxis was used only during hospitalization? This possible re-evaluation of the Finnish thromboprophylaxis recommendations is also supported by the recommendation of the European Society of Anaesthesiology for perioperative thromboprophylaxis after fast-track arthroplasty (27). The recommendation states that in selected cases of fast-track arthroplasty patients, thromboprophylaxis should be administered only during hospitalization.

Most of the operative site and all the intraocular bleedings in our study occurred during the use of thromboprophylaxis, suggesting that it might have some influence on these bleedings. For GIBs and ICHs, it is likely that the influence of thromboprophylaxis was minor only, as most of these potentially life-threatening bleedings occurred after termination of thromboprophylaxis. However, the arthroplasty population includes older patients with impaired renal function, a condition that could delay the clearance of anticoagulants. The use of non-steroidal pain medication might also have impaired renal function and consequently increased bleeding risk.

Our study has its weaknesses. 1st, the quality of the data collected depends on the quality of the register data. The completeness of the Finnish Arthroplasty Register for primary THA and TKA was over $90 \%$ during the years 2015 and 2016 (29). A systematic review of the quality of the Finnish Hospital Discharge Register (FHDR) evaluated its accuracy and coverage as ranging from satisfactory to very good (30). 2nd, the way hospitals record revisits or readmissions in the FHDR may vary. 3rd, the cause of discomfort in the operated joint may be recorded as pain, bleeding, swelling, or a suspected infection. These symptoms and findings may, alternatively, be recorded using the same diagnosis code as that of the primary operation, making it difficult to identify all clinically relevant bleeding complications. 4th, it is likely some bleeding complications were not filed in the FHDR. However, the incidence of life-threatening bleedings such as ICH and GIB or death due to bleeding is more reliably recorded in the FHDR due to their severity, as they often lead to reoperation, prolonged stay, or readmission.

We selected 2015 and 2016 as the study period to provide a reference point for future studies on the effects of changes made in the thromboprophylaxis protocol. 2 of the study hos- pitals have now changed their thromboprophylaxis protocol in line with the European Society of Anaesthesiology (ESA) recommendation. During the study period, all 3 hospitals were still following the Finnish thromboprophylaxis protocol.

A strength of our study is that the study population comprised an unselected sample of arthroplasty patients. Thus, we also included patients on permanent anticoagulation before surgery. Permanent anticoagulation is usually discontinued before surgery with or without bridging and continued after surgery or after the use of postoperative thromboprophylaxis. Bridging is used only in cases where the patient has a mechanical heart valve or a hereditary clotting disorder. Permanent anticoagulation is usually discontinued so that spinal anesthesia can be safely performed. The duration of discontinuation depends on the drug in question. If needed, platelet inhibitors are substituted for acetylsalicylic acid (ASA) and continued throughout surgery.

We also included patients who did not receive any anticoagulation due to an underlying illness. Their number was inconsequential, and they usually received mechanical thromboprophylaxis to prevent VTE. Thus, our study is representative of a fast-track arthroplasty population.

The rates of clinically relevant bleedings were similar between the THA, TKA, and BTKA procedures. This result conflicts with previous findings of more bleeding complications in TKA and BTKA than in THA procedures $(31,32)$ patients. 1 explanation for this difference might be that information on blood transfusions, blood loss during surgery, and change in hemoglobin is lacking in our study.

To conclude, the incidence of clinically relevant bleeding complications after fast-track arthroplasty was $1 \%$, and most of these were located at the operative site. One-third of these bleedings (intracranial and gastrointestinal bleedings) can be life-threatening, lead to hospitalization, or threaten independent living. In the fast-track setting studied here, clinically relevant bleeding complications were low.

AMM, KP, AE, JH, AK, PM, and JP wrote the manuscript. HK performed the data analysis. All contributed to the conception and design of the study, to critical analyses of the data, to interpretation of the findings, and to critical revision of the manuscript.

Acta thanks Henrik Husted for help with peer review of this study.

1. Rotondano G. Epidemiology and diagnosis of acute nonvariceal upper gastrointestinal bleeding. Gastroent Clin North Am 2014; 43(4): 643-63. doi: 10.1016/j.gtc.2014.08.001.

2. Strate L L. Lower GI bleeding: epidemiology and diagnosis. Gastroent Clin N Am 2005; 34(4): 643-64. doi: 10.1016/j.gtc.2005.08.007.

3. Adenikinju A S, Feng J E, Namba C A, Luthringer T A, Lajam C M. Gastrointestinal complications warranting invasive interventions following total joint arthroplasty. J Arthroplasty 2019; 34: 2780-4. doi: 10.1016/j.arth.2019.06.026. 
4. Falck-Ytter Y, Francis C W, Johanson N A, Curley C, Dahl O E, Schulman S, et al. Prevention of VTE in orthopedic surgery patients: antithrombotic therapy and prevention of thrombosis, 9th ed: American College of Chest Physicians evidence-based clinical practice guidelines. Chest 2012; 141: e278S-325S. doi: 10.1378/chest.11-2404.

5. Jenny J-Y, Bulaid $\mathbf{Y}$, Boisrenoult $\mathbf{P}$, Bonin $\mathbf{N}$, Henky $\mathbf{P}$, Tracol $\mathbf{P}$, et al. Bleeding and thromboembolism risk of standard antithrombotic prophylaxis after hip or knee replacement within an enhanced recovery program. Orthop Traumatol Surg Res 2020; 106: 1533-8. doi: 10.1016/j. otsr.2020.02.026.

6. Husted H, Otte K S, Kristensen B B, Ørsnes T, Wong C, Kehlet H. Low risk of thromboembolic complications after fast-track hip and knee arthroplasty. Acta Orthop 2010; 81(5): 599-605. doi: $10.3109 / 17453674.2010 .525196$.

7. Jørgensen C C, Jacobsen M K, Soeballe K, Hansen T B, Husted H, Kjaersgaard-Andersen P, et al. Thromboprophylaxis only during hospitalisation in fast-track hip and knee arthroplasty, a prospective cohort study. BMJ Open 2013; 3(e003965). doi: 10.1136/bmjopen-2013-003965.

8. Petersen P B, Kehlet H, Jørgensen C C, Lundbeck Foundation Centre for Fast-track Hip and Knee Replacement Collaborative Group. Safety of in-hospital only thromboprophylaxis after fast-track total hip and knee arthroplasty: a prospective follow-up study in 17,582 procedures. Thrombosis Haemostasis 2018; 118(12): 2152-61. doi: 10.1055/s-0038-1675641.

9. Dahl O E, Quinlan D J, Bergqvist D, Eikelboom J W. A critical appraisal of bleeding events reported in venous thromboembolism prevention trials of patients undergoing hip and knee arthroplasty. J Thrombosis Haemostasis 2010; 8(9): 1966-75. doi: 10.1111/j.1538-7836.2010.03965.x.

10. European Medicines Agency. Guideline on clinical investigation of medical products for prophylaxis of high intra- and post-operative venous thromboembolic risk [Internet] 2007 [cited 2021 Dec 24]. Available from: https://www.ema.europa.eu/en/documents/scientific-guideline/ guideline-clinical-investigation-medicinal-products-prophylaxis-highintra-post-operative-venous_en.pdf.

11. Schulman S, Anger S U, Bergqvist D, Eriksson B, Lassen M R, Fisher W. Definition of major bleeding in clinical investigations of antihemostatic medicinal products in surgical patients. J Thrombosis Haemostasis 2010; 8(1): 202-4. doi: 10.1111/j.1538-7836.2009.03678.x.

12. Official Statistics of Finland. Official Statistics of Finland [Internet]. [cited 2020 Sep 22]. Available from: https://pxnet2.stat.fi/PXWeb/ pxweb/fi/StatFin/StatFin_vrm_vaerak/statfin_vaerak_pxt_11ra.px/.

13. Pamilo K J, Torkki P, Peltola M, Pesola M, Remes V, Paloneva J. Reduced length of uninterrupted institutional stay after implementing a fast-track protocol for primary total hip replacement: register-based analysis of 4 hospitals and 3,193 replacements. Acta Orthop 2018; 89(1): 10-16. doi: 10.1080/17453674.2017.1370845.

14. Finnish Arthroplasty Association. Hyvä hoito lonkan ja polven tekonivelkirurgiassa [Internet] 2015 [cited 2021 Aug 15] 1-130 p. Available from: https://www.saply.fi/wp-content/uploads/2020/09/hyva_ hoito_lonkan_ja_polven_tekonivelkirurgiassa_2015_small.pdf.

15. Nordic Medico-Statistical Committee. NOMESCO classification of surgical procedures (NCSP), version 1.15. [Internet]. [cited 2018 Aug 15]. Available from: http://urn.kb.se/resolve?urn=urn:nbn:se:norden:org:diva-4620.

16. World Health Organization. Classification of diseases (ICD) [Internet] 10th revision [cited 2018 Aug 15]. Available from: https://www.who.int/ standards/classifications/classification-of-diseases.

17. Paavolainen $\mathbf{P}$, Hämäläinen $\mathbf{M}$, Mustonen $\mathbf{H}$, Slätis $\mathbf{P}$. Registration of arthroplasties in Finland. Acta Orthop Scand 2009; 62(Suppl. 241): 27-30. doi: 10.3109/17453679109155101.
18. Järvelin J, Haapakoski J, Mäkelä K. Hip and knee arthroplasties 2016; Lonkan ja polven tekonivelet 2016. Finnish Institute for Health and Welfare https://thl.fi/en/web/thlfi-en 2018 [cited 2020 Nov 5]. Available from: https://www.julkari.fi/bitstream/handle/10024/135941/Tr02_18. pdf? sequence $=10 \&$ is Allowed $=\mathrm{y}$.

19. Järvelin J, Haapakoski J, Mäkelä K. Hip and knee arthroplasties 2015; Lonkan ja polven tekonivelet 2015 [Internet]. Finnish Institute for Health and Welfare https://thl.fi/en/web/thlfi-en 2016 [cited 2020 Nov 5]. Available from: https://www.julkari.fi/handle/10024/131579.

20. Bohl D D, Sershon R A, Fillingham Y A, della Valle C J. Incidence, risk factors, and sources of sepsis following total joint arthroplasty. $\mathrm{J}$ Arthroplasty 2016; 31(12): 2875-9.e2. doi: 10.1016/j.arth.2016.05.031.

21. Kwong L M, Kistler K D, Mills R, Wildgoose P, Klaskala W. Thromboprophylaxis, bleeding and post-operative prosthetic joint infection in total hip and knee arthroplasty: a comprehensive literature review. Expert Opin Pharmacother 2012 ; 13(3): 333-44. doi: $10.1517 / 14656566.2012$.

22. Berg U, Bülow E, Sundberg M, Rolfson O. No increase in readmissions or adverse events after implementation of fast-track program in total hip and knee replacement at 8 Swedish hospitals: an observational before-and-after study of 14,148 total joint replacements 2011-2015. Acta Orthop 2018; 89(5): 522-7. doi: 10.1080/17453674.2018.1492507.

23. Pamilo K J, Torkki P, Peltola M, Pesola M, Remes V, Paloneva J. Fast-tracking for total knee replacement reduces use of institutional care without compromising quality. Acta Orthop 2018; 89(2): 184-9. doi: 10.1080/17453674.2017.1399643.

24. Saku S A, Madanat R, Mäkinen T J. Reasons and risk factors for ninety day re-admission following primary total knee arthroplasty in a high-volume centre. Int Orthop 2018; 42(1): 95-9. doi: 10.1007/s00264017-3676-y.

25. An S J, Kim J, Yoon B-W. Epidemiology, risk factors, and clinical features of intracerebral hemorrhage: an update. J Stroke 2017; 19(1): 3-10. doi: 10.5853/jos.2016.00864.

26. Nielsen C S, Jans Ø, Ørsnes T, Foss N B, Troelsen A, Husted H. Combined intra-articular and intravenous tranexamic acid reduces blood loss in total knee arthroplasty a randomized, double-blind, placebo-controlled trial. J Bone Joint Surg Am 2016; 98(10): 835-41. doi: 10.2106/ JBJS.15.00810.

27. Venclauskas L, Llau J V, Jenny J-Y, Kjaersgaard-Andersen P, Øivind $\mathrm{J}$. European guidelines on perioperative venous thromboembolism prophylaxis: day surgery and fast-track surgery. Eur J Anaesthesiol 2018; 35: 134-8. doi: 10.1097/EJA.0000000000000706.

28. Jørgensen C C, Petersen P B, Reed M, Kehlet H. Recommendations on thromboprophylaxis in major joint arthroplasty: many guidelines, little consensus? J Thrombosis Haemostasis 2019; 17(2): 250-3.

29. National Institute for Health and Welfare. Finnish Arthroplasty Register-ENDOnet [Internet] 2020 [cited 2020 Sep 22]. Available from: https://www.thl.fi/far/\#data/cphd.

30. Sund R. Quality of the Finnish Hospital Discharge Register: a systematic review. Scand J Public Health 2012; 40(6): 505-15. doi: 10.1177/1403494812456637.

31. Gromov K, Troelsen A, Otte K S, Ørsnes T, Husted H. Morbidity and mortality after bilateral simultaneous total knee arthroplasty in a fast-track setting. Acta Orthop 2016; 87(3): 286-90. doi: 10.3109/17453674.2016.1141631.

32. Sizer S C, Cherian J J, Elmallah R D K, Pierce T P, Beaver W B, Mont M A. Predicting blood loss in total knee and hip arthroplasty. Orthop Clin N Am 201; 46: 445-59. 5doi: 10.1016/j.ocl.2015.06.002. 


\section{Supplementary data}

Appendix. NOMESCO codes - Finnish version

NFB30: Primary total prosthetic replacement of hip joint not using cement

NFB40: Primary total prosthetic replacement of hip joint using hybrid technique

NFB50: Primary total prosthetic replacement of hip joint using cement

NFB60: Demanding prosthetic replacement of hip

NFB99: Other primary prosthetic replacement of hip joint

NGB20: Primary total prosthetic replacement of knee joint without patellar part -sliding prosthesis

NGB30: Primary total prosthetic replacement of knee joint without patellar part - connected prosthesis

NGB40: Primary total prosthetic replacement of knee joint with patellar part -sliding prosthesis

NGB50: Primary total prosthetic replacement of knee joint with patellar part - connected prosthesis 